\title{
La globalización y la nueva salud pública
}

\author{
Julio Frenk, MC, PhD, (1) Octavio Gómez-Dantés, MC, MSc. ${ }^{(2)}$
}

E s un gran honor dictar la conferencia magistral que honra la memoria de unos de los más grandes profesionistas mexicanos de la salud pública, el Maestro Miguel E. Bustamante.

Constituye un honor adicional dictar esta conferencia justamente en el congreso que coincide con la celebración del vigésimo aniversario de la creación del Instituto Nacional de Salud Pública (INSP). Desde su nacimiento el INSP se propuso ser un espacio abierto al intercambio de ideas no sólo entre sus propios investigadores, sino entre los miembros de toda la comunidad académica nacional e internacional. Por eso se iniciaron con gran éxito, desde el año mismo de la creación de este Instituto, los congresos nacionales de Investigación en Salud Pública. Y desde ese primer congreso se decidió que el espacio de mayor relevancia sería una conferencia magistral dictada en el marco de la sesión plenaria inaugural. Cuando se discutió el nombre de la conferencia no hubo el mínimo titubeo: había que honrar la memoria del Maestro Miguel E. Bustamante.

Las razones eran obvias. El Maestro Bustamante puede considerarse legítimamente como el padre de la salud pública moderna en México. Desde luego, este campo había tenido antes que él a figuras señeras de la investigación y la práctica, pero ellas provenían de disciplinas vecinas a la salud pública propiamente dicha. Bustamante fue el primer mexicano que obtuvo un doctorado en salud pública, otorgado por la Universidad Johns Hopkins en los años treinta. Por cierto que hoy, gracias en gran medida a la magnífica labor de este Instituto, el país puede presumir más de un centenar de doctores en salud pública.
No se hará aquí un recuento exhaustivo de los logros de Miguel Bustamante. Solamente se quisiera destacar que su proverbial generosidad benefició también -y de manera directa- al INSP. Al empezar su gestión al frente de la Secretaría de Salud, el Dr. Guillermo Soberón designó al Maestro Bustamante como uno de sus más cercanos asesores. Cuando el Secretario Soberón le encargó a uno de los que esto escribe la organización del Centro de Investigaciones en Salud Pública y después del Instituto, de inmediato lo encomendó a la tutela protectora del Santo Patrón de la Salud Pública, Miguel Bustamante. Él abrazó la causa de la renovación de la salud pública y fue una de las fuerzas que inspiraron la fundación del INSP hace 20 años.

Fue una pena que el Maestro no alcanzara a ver materializado el fruto de sus generosas orientaciones. Él murió el 4 de enero de 1986, un año antes del nacimiento del INSP, aunque muchas de sus ideas inspiradoras encontraron cuerpo en este Instituto que hoy cumple ya 20 años. Uno de los que esto escribe guarda como uno de sus tesoros más preciados un ejemplar del Boletín que la Secretaría de Salud publicaba en aquellos años para dar cuenta de la actividad institucional. Ahí se publicó la oración fúnebre que el Secretario Soberón leyó durante el homenaje de cuerpo presente que se rindió al Maestro Bustamante al día siguiente de su fallecimiento, en el patio central del histórico edificio de Lieja. Con su conocida sensibilidad, el Secretario le había indicado al entonces director del Centro de Investigaciones en Salud Pública que durante la lectura de esa oración estuviera haciendo guardia ante el féretro del Maestro. Con ello, Soberón quería dejar clara la línea directa que, como director del Centro de Investi-

Este documento fue presentado en el marco de la Conferencia Magistral Miguel E. Bustamante, durante el XII Congreso Nacional de Investigación en Salud Pública, celebrado en las instalaciones del Instituto Nacional de Salud Pública, en Cuernavaca, Morelos, del 7 al 9 de marzo de 2007.

(I) Fundación Bill y Melinda Gates.

(2) Consultor independiente.

Solicitud de sobretiros: Dr. Julio Frenk. Av. Universidad 655, col. Santa María Ahuacatitlán. 62508, Cuernavaca, Morelos, México. Correo electrónico: jfrenk@prodigy.net.mx 
gaciones en Salud Pública, lo vinculaba con el padre de la salud pública moderna. El dolor por la desaparición física del Maestro Bustamante fue mitigado por la convicción íntima de saberse heredero de la fecunda tradición que él había iniciado.

Podrán entender, pues, que no hubo duda sobre la importancia de que el INSP honrara la memoria del ilustre personaje cuyo nombre lleva la conferencia que hoy nos reúne. Y podrán ustedes entender el júbilo que produce constatar que esta conferencia, y el espléndido congreso del que forma parte, se hayan transformado, con el curso del tiempo y la intervención de tantas personas, en una auténtica tradición académica.

Fue un acierto de los organizadores, además, solicitar que esta conferencia se dedicara precisamente a la globalización y la salud pública. Es otra manera de honrar al Maestro Bustamante, pues a lo largo de su carrera él mostró un gran interés por la salud internacional. Apenas concluidos sus estudios doctorales, diseñó, fundó y dirigió, con el apoyo de la Fundación Rockefeller, las Unidades Sanitarias Cooperativas, que eventualmente dieron origen a los Servicios Coordinados de Salubridad. ${ }^{1,2}$ En 1947 participó en la Conferencia Sanitaria Internacional que redactó la Constitución de la Organización Mundial de la Salud (OMS). Durante la siguiente década fue Secretario General de la Oficina Sanitaria Panamericana.

El rostro de la salud internacional se ha transformado intensamente en la época actual. Hoy hay nuevos actores, nuevos debates y, sobre todo, desafíos novedosos. Los cambios han sido tan espectaculares que el propio término de "salud internacional" empieza a ser sustituido por el de "salud global".

Para explorar este nuevo terreno es importante analizar las tres tendencias que están influyendo de manera determinante en la salud global a principios del siglo XXI: a) la creciente transferencia internacional de riesgos y oportunidades para la salud; b) el mayor pluralismo en la arena de la salud internacional, con una acelerada multiplicación del número de actores, y c) el papel cada vez más crítico de la salud dentro de la agenda del desarrollo económico, la seguridad global y la democracia.

El argumento central de esta conferencia es que para enfrentar los retos y aprovechar las oportunidades que en materia de salud plantea la globalización, resulta necesario empezar por redefinir el concepto mismo de salud global. En particular, es indispensable distinguirlo del concepto de salud internacional, que en el siglo XX se identificó exclusivamente con las enfermedades transmisibles en los países en vías de desarrollo y con actividades de asistencia técnica. Lo que se requiere es "globalizar" el concepto de salud global para darle el contenido que la interdependencia del mundo actual demanda.

\section{La transferencia internacional de riesgos $y$ oportunidades para la salud}

La transferencia internacional de riesgos y oportunidades para la salud no es un fenómeno nuevo. Desde hace siglos, el comercio, la migración, las guerras y las conquistas han puesto en contacto a poblaciones de lugares apartados. Lo novedoso es la amplitud y profundidad de la integración. Como nunca antes en la historia de la humanidad, las consecuencias incluso de acciones aparentemente nimias que suceden en lugares distantes se manifiestan, literalmente, en el umbral de nuestros hogares.

La creciente proximidad del mundo se hace evidente en diversos procesos. El número de viajeros internacionales asciende a tres millones de personas diariamente. ${ }^{3}$ Los mercados financieros mueven alrededor de dos mil millones de dólares al día. ${ }^{3}$ Ya hay más de mil millones de usuarios de la Internet. ${ }^{4} \mathrm{El} \mathrm{mis-}$ mo movimiento antiglobalización se globalizó en 2001, cuando activistas de todo el mundo se encontraron en el primer Foro Social Mundial en Porto Alegre, Brasil. La versión 2006 de este foro, que se llevó a cabo en Caracas, Venezuela, reunió a activistas de 170 países.

No se pueden desdeñar las implicaciones de estos cambios para las condiciones salud de nuestras poblaciones ni para los sistemas de atención.

El caso más comúnmente citado de la desaparición de las fronteras de la salud es la propagación de las enfermedades infecciosas. Éste tampoco es un fenómeno novedoso per se.

La primera epidemia transnacional documentada se produce durante la Guerra del Peloponeso, en el siglo V antes de nuestra era. ${ }^{5,6}$ Según Tucídides, esta epidemia acabó con una cuarta parte de la población de Atenas y con sus ambiciones imperiales. En el año 542 de nuestra era, la llamada Plaga de Justiniano devastó el Imperio Romano. ${ }^{7}$ A mediados del siglo XIII una nueva epidemia de peste, conocida como Muerte Negra, produjo el deceso de 20 millones de personas sólo en el Viejo Continente. ${ }^{8}$ La colonización de Brasil y el Caribe en el siglo XVI se acompañó de la introducción del sarampión y la viruela, lo que casi condujo al exterminio de los pueblos indígenas. ${ }^{9}$ Esta situación obligó a la importación de esclavos de África Occidental, quienes trajeron consigo el paludismo y la fiebre amarilla al Nuevo Mundo. Más recientemente, la pandemia de influenza de 1918, que al parecer se originó en una granja de cerdos de Kansas, 
produjo alrededor de 50 millones de decesos en todo el mundo, cinco veces el número de muertes producidas en combate durante la Primera Guerra Mundial. ${ }^{10,11}$

Como puede constatarse, la presencia cosmopolita de las enfermedades transmisibles tiene una larga historia. Lo verdaderamente novedoso es la escala de lo que se ha dado en llamar "tráfico microbiano". El incremento sin precedentes de la migración, el comercio y los viajes internacionales genera miles de contactos potencialmente infecciosos. Así, en Estados Unidos se produjo, en los años ochenta, un brote de dengue como resultado de la importación del mosquito tigre, que se introdujo en ese país en un cargamento de neumáticos usados procedente de Japón. ${ }^{12}$ De la misma manera, en 1991 el agua contaminada de un barco originario de Asia produjo en Perú un brote de cólera que se convirtió en una epidemia continental en cuestión de semanas. ${ }^{13}$

La tuberculosis es otro problema reemergente. En el mundo hay alrededor de 9 millones de personas que sufren de esta enfermedad, la cual produce más de 2 millones de muertes anualmente. Varias razones explican su regreso. Una de ellas es, por supuesto, la pandemia de VIH/SIDA. Otras variables que influyen en el desarrollo de esta infección son el hacinamiento, la mala nutrición y la falta de atención a la salud, factores todos ellos comunes entre los socialmente marginados. En Rusia, por ejemplo, toda la población de las prisiones está en peligro de adquirir infecciones con cepas multirresistentes. Se ha calculado que de los tres mil prisioneros que se liberan anualmente en ese país, $80 \%$ están infectados con tuberculosis y muchos son portadores de cepas resistentes. ${ }^{14} \mathrm{El}$ impacto de este tipo de situaciones trasciende las fronteras nacionales. Así, en marzo de 1999 los diarios de los principales países del mundo comentaban el caso de 12 pasajeros infectados con una cepa mortal de tuberculosis que adquirieron en un vuelo de París a Nueva York. La fuente del brote había sido un turista ucraniano. ${ }^{15}$

Las últimas adiciones a la lista de epidemias globales son el síndrome respiratorio agudo severo, o SARS, y la influenza aviar. Esta última es todavía un peligro regional, pero hay especialistas que anticipan una pandemia con devastadoras consecuencias sanitarias, económicas y políticas.

Este pequeño recuento, además de darnos una idea de la magnitud del tráfico microbiano, nos sirve para aclarar dos falsas concepciones que frecuentemente nublan la discusión sobre el papel de las enfermedades infecciosas en la agenda global de la salud.

La primera de estas falsas concepciones es que las enfermedades transmisibles representan una especie de etapa inferior en la evolución de los patrones de enfermedad que se clasifican bajo el concepto de "tran- sición epidemiológica". En su formulación original, propuesta por Omran a principios de los años setenta, se veía a la transición epidemiológica como un movimiento lineal de las enfermedades transmisibles hacia los padecimientos no transmisibles. ${ }^{16}$ Sólo era cuestión de tiempo para que las sociedades se deshicieran de la carga de las infecciones.

Esta concepción simplificadora ha sido superada. Gracias en gran medida a los trabajos de investigación realizados desde los primeros años del Instituto Nacional de Salud Pública, hoy sabemos que la transición en salud no es un fenómeno simple, lineal y unidireccional, sino un proceso complejo, contradictorio y dinámico en el que se traslapan varias etapas y en el que las poblaciones experimentan verdaderas "contratransiciones" con el resurgimiento de infecciones previamente controladas. ${ }^{17}$

Más aún, la separación entre enfermedades transmisibles y no transmisibles es menos clara de lo que se pensó hace algún tiempo. Para empezar, algunas enfermedades originalmente clasificadas como no transmisibles han demostrado tener un origen infeccioso. Además, muchas de estas enfermedades o sus tratamientos debilitan al sistema inmune y dan origen a infecciones asociadas que a menudo son la causa precipitante de muerte.

En suma, las enfermedades infecciosas no pueden considerarse como un componente exclusivo de una etapa primitiva de la transición en salud, sino que deben verse como un ingrediente cambiante de todo patrón epidemiológico. Esta conclusión nos lleva a discutir la segunda falsa concepción: que las enfermedades infecciosas son sobre todo un problema de los países en vías de desarrollo.

Como hemos visto, incluso en las sociedades en las que el patrón epidemiológico lo dominan las enfermedades no transmisibles, las infecciones constituyen un acompañante común de estos padecimientos. Más aún, el grado de integración de nuestro mundo supone que ningún país puede aislarse de los riesgos que surgen en otras partes del planeta.

La aceleración de la diseminación de las enfermedades infecciosas se relaciona con cambios radicales en nuestro ambiente y estilos de vida, que han llevado a Arno Karlen a hablar de una nueva era biocultural. ${ }^{13}$ En efecto, para complicar todavía más las cosas, no son solamente las personas y los microbios quienes viajan de un país a otro; también lo hacen las ideas y los estilos de vida.

El tabaquismo y la obesidad son los mejores ejemplos de los riesgos emergentes ligados a la globalización que están imponiendo una doble carga a los sistemas de salud en el mundo, complicando aún más las inequidades en salud. Hoy en día, los problemas úni- 
camente de los pobres, como el paludismo o la tuberculosis, ya no son los únicos problemas de los pobres, quienes también sufren en mayor grado de las enfermedades no transmisibles. Así, las muertes relacionadas con el consumo de tabaco se están concentrando de manera creciente en los países en vías de desarrollo, los cuales carecen de las estructuras regulatorias para confrontar el enorme poder de las empresas multinacionales. La manera de oponerse a ese poder es complementando las políticas nacionales con instrumentos globales, como el Convenio Marco para el Control del Tabaco, que constituye el primer tratado internacional de salud pública. Con orgullo se puede mencionar que México fue el primer país de la región de las Américas que firmó dicho convenio.

Pero la globalización va más allá de las enfermedades y los riesgos para incluir también a los productos para la salud. El creciente comercio de servicios y medicamentos a través de la Internet, por ejemplo, tiene enormes implicaciones para la prescripción irracional y la consecuente diseminación de resistencias microbianas. El hecho de que no se trata ya de un fenómeno marginal queda demostrado con los esfuerzos recientes de la OMS por controlarlo.

El movimiento transfronterizo de los proveedores de servicios de salud también se ha incrementado de manera importante. El ejemplo más dramático es la migración de enfermeras, cuya escasez periódica es un fenómeno común en diversos países desarrollados, de manera particular en Estados Unidos. Durante los últimos 15 años este problema se ha enfrentado mediante la importación de enfermeras de Filipinas, Jamaica, Nigeria, Kenia, la India y los países de habla inglesa del Caribe, y ahora también de México. Este fenómeno migratorio a menudo debilita los sistemas de salud de los países exportadores. La tendencia se está agudizando dado el déficit de enfermeras que se anticipa para la próxima década. Recientemente el Congreso de Estados Unidos reservó 50000 visas de inmigración para enfermeras. ${ }^{18}$

Los riesgos y las oportunidades inherentes a este proceso son considerables y requieren de un examen cuidadoso. El debate internacional sobre las responsabilidades de todos los actores ha generado propuestas interesantes, dentro de las que se incluyen el diseño de guías éticas para el reclutamiento del personal de salud y la compensación financiera a los países exportadores. ${ }^{19}$

\section{Los actores de la salud global}

Además de la transferencia internacional de riesgos y oportunidades para la salud, el otro proceso que está influyendo de manera importante en la salud global es la proliferación de actores.
Hasta la fundación de la Organización Mundial de la Salud (OMS) en 1948, las instituciones internacionales en este campo no sólo eran escasas, sus actividades eran muy limitadas. ${ }^{20}$ Producto de la Segunda Conferencia Internacional de Estados Americanos celebrada en la ciudad de México en 1901, la Oficina Sanitaria Panamericana dedicó sus primeras décadas de existencia a generar información sobre epidemias de importancia regional. Por su parte, la Oficina Internacional de Higiene, fundada en 1907, se limitó a desarrollar actividades de registro de estadísticas vitales y vigilancia epidemiológica, sobre todo de aquellas enfermedades que podían representar una amenaza para los países de Europa Occidental. La Organización de Salud de la Liga de las Naciones, formada en 1923, terminó desarrollando actividades similares también en beneficio, sobre todo, de los países europeos. Este elenco lo completaban unas cuantas organizaciones privadas filantrópicas, dentro de las que destaca la Fundación Rockefeller. ${ }^{21}$

La OMS absorbe y unifica a todas las organizaciones multilaterales existentes en un solo cuerpo intergubernamental con amplias responsabilidades -ya no sólo el control de la diseminación internacional de enfermedades infecciosas-, y con el poder para implantar regulaciones y adoptar convenciones y acuerdos.

En la segunda mitad del siglo XX, además de la OMS, otras agencias y programas del sistema de las Naciones Unidas empiezan a desarrollar actividades de salud, en particular UNICEF, PNUD, FAO y UNESCO. Durante este periodo también hacen su aparición las agencias de cooperación bilateral de los países industrializados.

A partir de la década de los años noventa, el número de actores de la salud internacional se incrementa de manera espectacular. Entre ellos destacan los bancos multilaterales de desarrollo, que han alcanzado una presencia financiera y técnica considerable. El Informe sobre el Desarrollo Mundial: Invirtiendo en Salud y el libro Prioridades para el Control de Enfermedades en los Países en Desarrollo, publicados por el Banco Mundial en esa década, constituyen verdaderos hitos en la historia reciente de la salud internacional. ${ }^{22,23}$ Con mucha satisfacción podemos decir que uno de los editores de esas dos importantes publicaciones fue otro brillante sanitarista mexicano, José Luis Bobadilla, investigador fundador del INSP, cuya memoria también se honra con una conferencia magistral en estos congresos.

Las organizaciones filantrópicas dedicadas a la salud también han proliferado y juegan ya un papel central. Aquí destaca la Fundación Bill y Melinda Gates, cuyo patrimonio habrá de crecer durante los próximos años a más de 60 mil millones de dólares, con lo cual sus donativos estarán sumando 3500 millones de 
dólares anuales, casi el triple del presupuesto de la OMS. ${ }^{24}$

Diversas instituciones académicas -sobre todo de los países industrializados pero crecientemente también de los países en desarrollo, como lo demuestra el INSP- se cuentan asimismo dentro de los actores de este campo. Además de generar conocimientos y de formar a muchos de los profesionales de la salud pública de todo el mundo, estas instituciones están participando de manera creciente en la definición de la agenda de la salud global.

Las organizaciones no gubernamentales, por su parte, cabildean a favor de ciertos temas, defienden activamente los intereses de diversos grupos, llevan a cabo tareas de investigación e información, y operan programas y servicios de todo tipo. Su influencia ha crecido a tal grado que analistas como Jessica Mathews incluyen a estas organizaciones dentro de los principales responsables de lo que ha llamado "desplazamiento del poder" del Estado a la sociedad civil..$^{25}$ Estas organizaciones han jugado un papel central en varios de los temas más controvertidos de la agenda mundial de la salud, como el acceso a medicamentos y los derechos de propiedad intelectual. Según cálculos recientes, sólo en el campo del VIH/SIDA, hay más de $60 \mathrm{mil}$ organizaciones no gubernamentales en el mundo.

Finalmente, tenemos a las compañías multinacionales responsables de la producción y distribución de la mayor parte de los bienes relacionados con la salud. Dado el ritmo al que se están consolidando, dichas empresas están incrementando su nivel de influencia en el mundo de la salud internacional.

A partir de esta diversificación institucional han empezado a surgir formas novedosas de alianzas público-privadas entre las agencias tradicionales del sistema de Naciones Unidas, los bancos multilaterales, las fundaciones filantrópicas, las organizaciones civiles e incluso algunas empresas multinacionales. El resultado es una pléyade de lo que podríamos llamar organismos "cuasi-multilaterales". El primero de ellos fue la Alianza Global para las Vacunas y la Inmunización (GAVI, por sus siglas en inglés) que hoy representa la principal fuente de financiamiento en su campo. Otro destacado integrante de este novedoso elenco institucional es el Fondo Global para la Lucha contra el SIDA, la Tuberculosis y la Malaria, que en los escasos cinco años desde su creación ha desarrollado una cartera de proyectos de apoyo por cerca de $7 \mathrm{mil}$ millones de dólares.

El gran reto en este escenario de enorme pluralismo es desarrollar un verdadero sistema global de salud que permita generar acciones concertadas en materia de definición de prioridades, movilización de recur- sos, diseño e implantación de estrategias, y monitoreo y evaluación de resultados.

\section{La salud en la agenda global}

El tercer proceso que está definiendo la salud global es su creciente importancia dentro de la agenda de los grandes temas de nuestros tiempos. La salud, de hecho, se está moviendo de los dominios de la llamada "política menor", que se relaciona con los asuntos humanitarios, al reino de la "política mayor", que está relacionada con la seguridad, el crecimiento económico y el poder. ${ }^{26}$

En diversos informes internacionales se ha incluido a ciertas enfermedades infecciosas dentro de una compacta lista de amenazas con las que el mundo debe lidiar ahora y en las décadas por venir. ${ }^{27,28}$ Estas amenazas no reconocen fronteras y deben encararse en los planos global, regional y nacional. Ningún estado, por más poderoso que sea, podría por sí solo volverse invulnerable a ellas.

Además del VIH/SIDA, la tuberculosis, el SARS y la gripe aviar, comentadas anteriormente, la seguridad global puede verse amenazada por el bioterrorismo. Mucho se ha discutido sobre la posibilidad de que se den este tipo de ataques y sobre su magnitud. Lo que parece claro, sin embargo, a la luz de los sucesos de Nueva York, Madrid y Londres, y el rápido crecimiento del poder de la biotecnología, es la necesidad de fortalecer nuestros sistemas de vigilancia a través de la creación de redes internacionales de laboratorios de salud pública y el diseño de mecanismos eficientes de intercambio de información. Independientemente de que se materialice o no algún ataque, tales medidas en sí mismas pueden mejorar el funcionamiento cotidiano de nuestros sistemas de salud.

Además de su identificación como componente central de la seguridad global, la percepción sobre el papel de la salud en el desarrollo se ha modificado en años recientes. Durante décadas se consideró la salud como un asunto básicamente humanitario. Gracias a la investigación económica hoy sabemos que la salud forma parte del capital humano, favorece el aprendizaje, aumenta la productividad, ayuda a combatir la pobreza y alienta el crecimiento económico. Todas estas razones han hecho que diversos analistas hablen de lo que han denominado "desarrollo guiado por la salud" o "health-led development". ${ }^{29}$

El influyente informe de la Comisión de Macroeconomía y Salud, establecida por la Dra. Gro Harlem Brundtland durante su gestión al frente de la OMS, logró demostrar que la salud no es sólo una consecuencia del nivel de desarrollo económico, sino tam- 
bién uno de sus principales determinantes. ${ }^{30}$ México forma parte de un selecto grupo de países pioneros que siguieron la recomendación de la Comisión global y establecieron instancias nacionales para examinar dicha relación en sus respectivos contextos. Es motivo de enorme satisfacción que el informe final de la Comisión Mexicana de Macroeconomía y Salud vaya a presentarse en el marco de este Congreso.

Hay un fenómeno emergente en la relación entre salud y desarrollo económico. Se trata de lo que hemos llamado la "paradoja inaceptable". ${ }^{31}$ Sabemos que la salud es una de las maneras más efectivas de promover el bienestar económico y combatir la pobreza. Sin embargo, la misma atención a la salud puede convertirse en un factor de empobrecimiento para muchos hogares cuando un país carece de los mecanismos sociales que garantizan un financiamiento justo de la atención a la salud. Se calcula que al año por lo menos 100 millones de hogares incurren en gastos catastróficos por motivos de salud en todo el mundo. ${ }^{32}$ Uno de los principales retos de los países de ingresos bajos y medios es garantizar la protección financiera universal en materia de salud, como lo está haciendo México mediante el Seguro Popular, el cual ha atraído la atención de muchas otras naciones interesadas en introducir innovaciones similares.

Por último, la promoción de la democracia y los derechos humanos es un tema más de la agenda global donde la salud está adquiriendo relevancia creciente. Se sabía que las sociedades democráticas tienden a contar con sistemas de salud que garantizan el acceso universal a servicios de salud. El mejor ejemplo posiblemente sea el Servicio Nacional de Salud del Reino Unido. Poco, sin embargo, se sabía sobre el efecto específico de ciertos principios democráticos en las condiciones de salud de las poblaciones.

Amartya Sen ha argumentado que las prácticas democráticas ayudan a prevenir los grandes desastres sociales, como las hambrunas o las epidemias. ${ }^{33,34}$ La libertad de prensa permite llamar la atención de la opinión pública hacia las necesidades de la gente, mientras que las elecciones democráticas obligan a los partidos en el poder a justificar sus políticas o modificarlas para atender mejor las demandas de la población. Lo contrario sucede en las sociedades autoritarias. El mejor ejemplo reciente es la censura gubernamental de la información sobre la diseminación del SARS en la provincia china de Guangdong en 2003, que aceleró la propagación de esta enfermedad al resto del país y del mundo.

Un estudio publicado en el British Medical Journal, que utilizó información de 170 países, mostró un efecto positivo de la democracia sobre la salud, que se mantiene después de ajustar por riqueza, nivel de desigualdad y magnitud del sector público de los países estudiados. ${ }^{35}$

El efecto inverso, el de la salud sobre la democracia, también empieza a llamar la atención. En un informe sobre la democracia en América Latina realizado por el PNUD (Programa de las Naciones Unidas para el Desarrollo), se recomienda extender el derecho a la atención a la salud para ampliar la efectividad de nuestras democracias. ${ }^{36}$

Marshall, en su obra seminal sobre Clase, ciudadanía y desarrollo social, ya había afirmado que el desarrollo de la ciudadanía culmina con la implantación de los derechos sociales, dentro de los que se incluye el derecho a la atención a la salud. ${ }^{37}$

Estas ideas han tenido un gran eco en México. El Programa Nacional de Salud 2001-2006, que se subtitula "La democratización de la salud en México", señaló la necesidad de universalizar el ejercicio real del derecho a la protección de la salud. Esta propuesta se concretó con la aprobación de la reforma a la Ley General de Salud de 2003 que dio origen al Sistema de Protección Social en Salud y su brazo operativo, el Seguro Popular. No cabe duda que al democratizar la atención a la salud, los mexicanos elevarán el nivel de bienestar y, al mismo tiempo, se estará contribuyendo a mejorar la salud de la democracia en el país.

\section{La globalización de la salud global}

Dada su creciente relación con áreas críticas de la política internacional, la salud puede ahora promoverse no sólo como un objetivo deseable en sí mismo, sino también como una fuente de seguridad global, un determinante del desarrollo y un instrumento para el buen gobierno. Sin embargo, para que esta visión pueda materializarse es necesario renovar el pensamiento y la acción en materia de cooperación internacional en salud. Si hoy hay más interés que nunca en la salud global, debemos empezar por tener una clara concepción sobre lo que ese término significa y cómo se distingue de otros similares.

Desde que se acuñó, alrededor de $1913^{38}$ el término "salud internacional" se identificó casi exclusivamente con el control de epidemias en puertos y fronteras, y con las necesidades de salud de los países pobres, que se asumían como amenazas. No es de extrañar, por lo tanto, que las actividades de salud internacional se catalogaran como asistencia y defensa, y se diseñaran e implantaran a través de perspectivas unilaterales.

La agenda de la salud internacional también se vio influida por la idea de que la mayor parte de las necesi- 
dades de salud podría enfrentarse a través de intervenciones técnicas y no como problemas que tienen fuertes componentes conductuales, culturales, políticos y económicos. El corolario de esta manera de pensar fue la definición de las prioridades en salud en términos estrictamente médicos y la inclusión en la agenda únicamente de aquellos problemas que se prestaban a soluciones tecnológicas. Esto era reflejo de la convicción de los años cincuenta y sesenta de que la ciencia y las habilidades administrativas de los países desarrollados bastaban para transformar al mundo en desarrollo. ${ }^{39}$

Durante la segunda mitad del siglo XX, la descolonización e independencia de varios países asiáticos y africanos, el creciente poder de las agencies multilaterales y las ONGs internacionales, y la aparición de nuevas teorías sobre el desarrollo, equilibraron la influencias de aquellas visiones etnocéntricas.

Sin embargo, en años recientes una especie de modernización lingüística ha revitalizado esta forma de pensar mediante el uso y la difusión del concepto de "salud global", un término que fue utilizado por vez primera vez en los años cincuenta. ${ }^{38}$ Los contenidos que se le han dado a este término nos recuerdan los que se le atribuyeron a la salud internacional en el siglo pasado.

En los medios de comunicación, la literatura académica y diversas iniciativas, la salud global se identifica con problemas que supuestamente son característicos del mundo en desarrollo, y la cooperación internacional con una especie de "voluntarismo tecnológico" mediante el cual la mera voluntad política y, sobre todo, financiera de desarrollar nuevas soluciones técnicas bastaría para resolver dichos problemas. Los beneficiarios de esta asistencia son presentados, en palabras de Alan Finkielkraut, como seres dependientes, enteramente penetrados por el sufrimiento, la enfermedad y la necesidad, y carentes de voluntad. ${ }^{40}$ De nuevo, lo que prevalece en este uso sesgado del término "salud global" es la idea de las sociedades pobres, ignorantes, pasivas y tradicionales que requieren de la caridad y la tecnología de los países ricos.

$\mathrm{Al}$ igual que el viejo concepto de salud internacional, la salud global ha puesto demasiado énfasis en los programas dedicados a atender enfermedades particulares, sobre todo de naturaleza infecciosa, y se ha olvidado de nueva cuenta de fortalecer los sistemas de salud. Como menciona Laurie Garrett, muchas mujeres infectadas por el VIH están recibiendo medicamentos para controlar su enfermedad y prevenir la transmisión del virus a sus bebés, pero no cuentan con acceso a la más rudimentaria atención del parto ni a vacunas para sus hijos. ${ }^{41}$ Más aún, muchos de los pacientes con VIH/ SIDA que reciben antirretrovirales de manera gratuita se atienden en unidades que no cuentan con los recursos humanos, el equipo y los insumos para darle un seguimiento adecuado a su tratamiento, lo que explica en buena medida la aparición de resistencias. ${ }^{42}$ “¿De qué nos sirven todas los medicamentos y vacunas del mundo si no hay nadie en las unidades para administrarlas?", se pregunta Barry Bloom, Director de la Escuela de Salud Pública de Harvard. ${ }^{43}$ Necesitamos balas mágicas, es cierto, pero también requerimos de "pistolas mágicas". ${ }^{44}$ Esas pistolas son los sistemas de salud. Nosotros agregaríamos que también necesitamos "gatilleros mágicos", esto es, recursos humanos capaces de planear y operar los sistemas de atención.

A nuestro juicio, estas limitaciones de los contenidos del término salud global y de la práctica a ella asociada nos obligan "globalizarlo" para reflejar de mejor manera la realidad actual. Y aquí, para explicarnos, permítanos recurrir a términos que se utilizaron en la discusión de lo que se dio en llamar la "nueva salud pública", concepto que proporcionó el marco de referencia para la organización del Instituto Nacional de Salud Pública. ${ }^{45}$

Podríamos empezar por definir la salud global y reconocerla como un campo de conocimiento y como un ámbito para la acción. Al igual que la nueva salud pública, la nueva salud global se define en términos de su nivel de análisis, que es el nivel poblacional. ${ }^{46}$ Su característica distintiva es el tipo de población, que son los miembros de la comunidad mundial: las naciones, los estados, las agencias multilaterales, las instituciones filantrópicas, las corporaciones trasnacionales, los centros académicos y las organizaciones de la sociedad civil.

Como campo de conocimiento, la salud global supone el estudio interdisciplinario del proceso de salud-enfermedad en el nivel mundial y de las respuestas sociales que se generan para enfrentar dicho proceso.

Por lo que respecta al proceso de salud-enfermedad, el concepto que mejor corresponde al nivel mundial de análisis es el de "transferencia internacional de riesgos", sean estos ambientales, infecciosos, o derivados de los estilos de vida, tal como fue explicado antes. En el corazón de este concepto yace la idea de la interdependencia y la complejidad de las condiciones de salud globales: el hecho de que muchos problemas son comunes a todos los países; no son exclusivamente de naturaleza transmisible; se diseminan a través de los canales creados para sostener el movimiento internacional de personas y bienes, y no sólo involucran la transferencia de riesgos desde los países pobres hacia los ricos, sino también en la dirección opuesta. En este sentido, Lincoln Chen y colaboradores han hablado de 
una era de "interdependencia global en salud" paralela a la era de "interdependencia económica". ${ }^{47}$

La respuesta social ante tal interdependencia se articula a través del sistema de salud mundial integrado por la pluralidad de actores que fueron analizados antes. Por ello, en tanto que campo del conocimiento, la salud global debe comprender el análisis de la arquitectura institucional para responder a la creciente complejidad creada por la transferencia internacional de riesgos.

Como ámbito para la acción, la salud global comprende el esfuerzo sistemático para identificar las necesidades de salud de la comunidad global y la organización de respuestas entre los miembros de esta comunidad para enfrentar dichas necesidades, incluyendo la formulación de políticas, la movilización de recursos y la implantación de estrategias. Mientras que la salud internacional tradicional se preocupó por la asistencia técnica de los países desarrollados a los países en desarrollo, la nueva salud global enfatiza la cooperación entre todos los actores en materia de educación, investigación y prestación de servicios de salud.

El imperativo de la cooperación radica en lo que podría denominarse la "paradoja de la soberanía". 48 En un mundo donde la unidad de organización política sigue siendo el Estado nacional, la responsabilidad por la salud de cada población recae en el gobierno respectivo. Sin embargo, como hemos analizado a lo largo de esta conferencia, un número creciente de determinantes de la salud está vinculado con la interdependencia entre los países y por lo tanto queda más allá del control de cualquier gobierno individual. La única forma de resolver esta paradoja es a través de la acción colectiva internacional, por medio de la cual los estados nacionales comparten su soberanía para alcanzar objetivos que ninguno puede lograr por sí solo. Entre esos objetivos la salud es el más trascendente para avanzar hacia el desarrollo, la seguridad, la democracia y el valor universal de los derechos humanos.

\section{Conclusión}

Estamos en un momento único de retos y oportunidades. Debido a la interdependencia, los retos a la salud exhiben una complejidad sin precedentes. Pero al mismo tiempo, nunca había habido tanto interés por la salud en los foros mundiales y los recursos para financiar programas han crecido de manera exponencial durante la última década. Hay un nuevo optimismo global sobre la posibilidad real de lograr mejoras sensibles en la salud de las poblaciones más pobres del mundo.

Para que el optimismo no se torne en decepción, se debe renovar tanto el concepto de salud global como su práctica mediante el impulso incluyente a la cooperación entre todos los actores del pluralista escenario mundial.

Un hombre que en su momento supo renovar el pensamiento y la acción fue Miguel E. Bustamante. Hoy debemos seguir el camino por él marcado del compromiso infatigable con la salud de cada uno de los habitantes de esta casa común que es nuestro mundo interdependiente.

\section{Referencias}

I. Palomar de Miguel J. Bustamante ME. En: Palomar de Miguel J.

Diccionario de México. México, DF:Trillas, 2005:211.

2. Bustamante ME. Enciclopedia de México, Tomo II. México, DF: Enciclopedia de México, 1978:178.

3. Kearney AT, Foreign Policy. Midiendo la globalización. Este País 200I;(I 22):2-9.

4. Kearney AT, International Endowment for Peace. Measuring globalization. Foreign Policy 2004;2:54-69.

5. Rosen G.A history of public health. Baltimore:The Johns Hopkins University Press, 1993:6.

6. Porter R. Blood and guts. A short history of medicine. Nueva York: WW Norton \& Company, 2002:8.

7. Porter R, ed. Medicine. Cambridge illustrated history. Cambridge: Cambridge University Press, 1996:28.

8. Porter R, ed. Medicine. Cambridge illustrated history. Cambridge: Cambridge University Press, 1996:25.

9. Porter D. Health, civilization and the state.A history of public health from ancient to modern times. Londres y Nueva York: Routledge, 1999:47. 10. Kolata G. Flu. The story of the great influenza pandemic of 1918 and the search for the virus that caused it. Nueva York: Farrar, Straus and Giroux, 1999:9.

I I. Kunstler JH. The long emergency. Nueva York: Grove Press, 2005: I I. 12. Hawley WA. Aedes albopictus in North America: probable introduction in used tires from northern Asia. Science 1987; 236: I I |4- I I I6.

13. Karlen A. Man and microbes. Disease and plagues in history and modern times. Nueva York: Simon and Schuster, 1995:216.

14. Remnik D. More bad news from the Gulag. The New Yorker 1999 February 15:27-28.

15. York G. A deadly strain of TB races toward the West. The Globe and Mail 1999 March 24:AI and AI2.

16. Omran AR. The epidemiologic transition: a theory of the epidemiology of population change. Milbank Mem Fund Q 1971;49:509-538.

17. Frenk J, Bobadilla JL, Sepúlveda J, López-Cervantes M. Health transition in middle-income countries: new challenges for health care. Health Pol Plann 1989;4:29-39.

18. Garret L. The challenge of global health. Foreign Affairs 2007;86(I): 14-38.

19. Brush B, Sochalski J, Berger A. Imported care: recruiting foreign nurses to US health care facilities. Health Affairs 2004;23(5):78-87. 20. Gómez-Dantés O. Health. En: Simmons PJ, De Jonge Oudraat C, eds. Managing global issues. Lessons learned. Washington, DC: Carnegie Endowment for International Peace, 2001:392-323.

2 I. Birn AE. Marriage of convenience. Rockefeller international health and revolutionary Mexico. Rochester: Rochester University Press, 2006. 22.World Bank.World Development Report 1993. Investing in health. Nueva York: Oxford University Press, 1993.

23. Jamison DT, Mosley WH, Meacham AR, Bobadilla JL, eds. Disease control priorities in developing countries. Nueva York: Oxford University Press, 1993. 
24. Fleishman JL. The foundation: a great American secret. Nueva York: Public Affairs, 2007.

25. Mathews J. Power shift. Foreign Affairs 1997;76(I):50-66.

26. Fidler DP. Health and foreign policy: a conceptual overview. Londres: The Nuffield Trust, 2005.

27. United Nations High Level Panel on Threats, Challenges and Change. A more secure world: our shared responsibility. Nueva York: Naciones Unidas, 2004

28. Commission on Human Security. Human security now: protecting and empowering people. Nueva York: Commission on Human Security, 2003.

29. Bloom DE, Canning D. The health and wealth of nations. Science 2000;287:12-13.

30. Commission on Macroeconomics and Health. Macroeconomics and health: investing in health for economic development. Ginebra: OMS, 200 I.

3I. Frenk J. Bridging the divide: global lessons from evidence-based health policy in Mexico. Lancet 2006; 368:954-96I.

32. Xu K, Evans D, Carrin G,Aguilar-Rivera AM. Designing health financing systems to reduce catastrophic health expenditure. Ginebra; WHO, Technical Briefs for Policy Makers No.2, 2005.

33. Sen AK. Development as freedom. Nueva York: Albert Knopf, 1999. 34. Ruger JP. Democracy and health. QJM 2005;98:299-304.

35. Franco A, Álvarez-Dardet C, Ruiz MT. Effect of democracy on health: ecological study. BMJ 2004;329:142I-I424.

36. Programa de las Naciones Unidas para el Desarrollo. La democracia en América Latina. Hacia una democracia de ciudadanas y ciudadanos. Lima, Perú: PNUD, 2004.
37. Marshall TH. Class, citizenship and social development. Nueva York: Doubleday Anchor Books, 1965.

38. Brown TM, Cueto M, Fee E. The World Health Organization and the transition from international to global public health. Am J Public Health 2006;96(I):62-72

39. Tendler J. Inside foreign aid. Baltimore:The Johns Hopkins University Press, 1975.

40. Beck H. Caridad global. Letras Libres 2006;(88):30-31.

4I. Garret L. The challenge of global health. Foreign Affairs 2007;86(I):14-38.

42. Epstein H, Chen L. Can AIDS be stopped? New York Rev Books 2002;49(4).

43. Bloom B. Public health in transition. Sci Am 2005;283(3):92-99.

44. Specter M. What money can buy. New Yorker 2005;October 24:57-71. 45. Frenk J. La salud de la población. Hacia una nueva salud pública. México, DF: FCE, 1994.

46. Frenk J, Chacón F. Conceptual bases for education and research in international health. En: Pan American Health Organization. International health. A North-South debate. Washington, DC: PAHO, 1992:205-221.

47. Chen L, Bell D, Bates L. World health and institutional change. En: Pocantico Retreat. Enhancing the performance of international health institutions. Cambridge, MA:The Rockefeller Foundation, Social Science Research Council, Harvard School of Public Health, 1996:9-21.

48. Jamison DT, Frenk J, Knaul F. International collective action in health: Objectives, functions and rationale. Lancet 1998;351:5 |4-5 I7. 